tylopora, and Uteria. This group at present contains over fifty genera, which are for the most part to be met with in the triassic, jurassic, cretaceous, and tertiary strata. In the number of those actually living there is a notable falling off, there being not more than the seven following genera :-Dasycladus, Halicoryne, Cymopolia

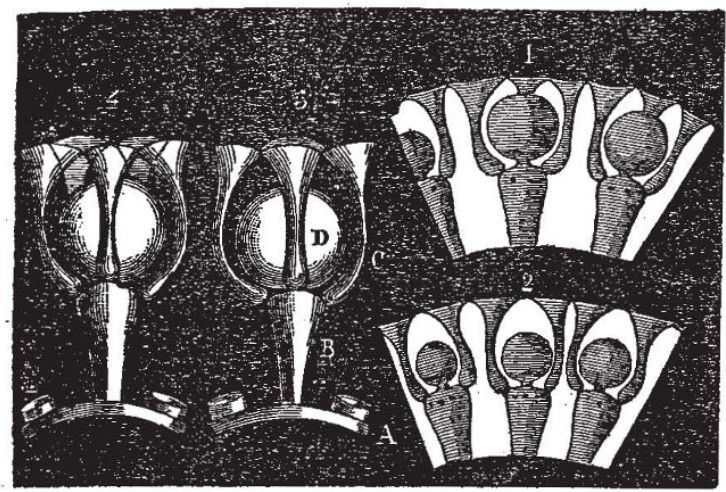

Fig. x.-Transverse section of a morsel of the calcarecus tube of $C_{y}$ mopolia rosarium, Lamr., showing the canals which receive the wherl of cel lules and the central spcrangial cavity. Fig. 2.- Tiansverse section of

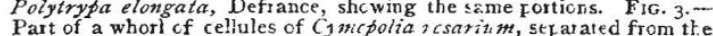
calcareous tute by acid. A, wall of celtral cellule; $\mathrm{k}$, first row of cellules; $c$, terminal whorl i $f$ cellules, in the centre of which is $D$, the axillary sperangium. FIG. 4.-Exactly the same farts in Polytrypa elongata, ottained from a mculd.

(with two sub-genera, Polytrypa and Decaisnella, ${ }^{2}$ g.n.), Polyphysa, Acetabularia, Neomeris, and Bornetella," g.n." [Doubtless a few more genera of recent forms yet remain to be described. Thus Chloroclados, of Sonder, appears to be a good and distinct genus allied to Dasycladus.]

"The frond in the Siphonea verticillata is simple or dichotomous; it consists of a central tubular unicellular axis, around which are arranged the radiary and verticellate ramuli, the exact arrangement of which varies according to the genera and to the species. In most of the species carbonate of lime is found deposited in abundance in the outer walls of the main axis and its ramuli, and this forms around the plant a calcareous envelope, in which is reproduced all the details of its organisation. This mineral coating may consist of one or of two calcareous cylinders. The inner cylinder will be formed by the central axis, and the first row of cells ${ }^{-}$which arise therefrom. The outer cylinder is laid down by the most external of the verticels of cells; these terminate by a splayed-out enlargement, the lateral edges of which become more or less consolidated with the similar enlargements of neighbouring cells, and by thus causing a reciprocal pressure, very regular hexagonal surface markings are produced. The organs of fructification are themselves surrounded by calcareous material, and assist in the formation of the outer cylinder, a fact easily seen in any section of Cymopolia."

The result of such an organisation is that when the organic vegetable matter becomes destroyed there still remains in those fossil species, which laid down a great deal of calcareous material, as well as in those living species-which lay down more or less of it-a skeleton permeated by canals (rays of the ramuli) and chambers (fructification). This arrangement, which permits of an exact classification of the fossil species, being wrongly interpreted, led even some most distinguished authors to see in these morsels of plants the full organisation of a Foraminifer."

Space will not permit of the table of the tuenty-two genera and seven families as detailed in the Comptes Rencius

I Type, Dactyiopora eruca, Paker.
2 Type, Neomeris nitida, Harv. MS. being here given, but every botanist will look forward with interest to the promised future detailed contributions of the author on this subject. Here it seems desirable to add that his conclusions are in every particular acquiesced in by one in every way thoroughly able to judge of the facts, Dr. Ed. Bornet, and having written this I feel it almost superfluous to say that on a careful study myself of specimens prepared by M. Munier-Chalmasfor which I take this opportunity of thanking him-I cannot conceive his demonstration as admitting of a doubt.

ED. Perceval Wright

\section{ELECTRICITY AND WATER DROPS I}

$\mathrm{T}$ has been known for many years that electricity has an extraordinary influence upon the behaviour of fine jets of water ascending in a nearly vertical direction. In its normal state a jet resolves itself into drops, which even before passing the summit, and still more after passing it, are scattered through a considerable width. When a feebly electrified body is brought into its neighbourhood, the jet undergoes a remarkable transformation, and appears to become coherent; but under more powerful electrical action the scattering becomes even greater than at first. The second effect is readily attributed to the mutual repulsion of the electrified drops, but the action of feeble electricity in producing apparent coherence has been a mystery hitherto.

It has been shown by Beetz that the coherence is apparent only, and that the place where the jet breaks into drops is not perceptibly shifted by the electricity. By screening various parts with metallic plates, Beetz further proved that, contrary to the opinion of earlier observers, the seat of sensitiveness is not at the root of the jet where it leaves the orifice, but at the place of resolution into drops. As in Sir W. Thomson's water-dropping apparatus for atmospheric electricity, the drops carry away with them an electric charge, which may be collected by receiving the water in an insulated vessel.

I have lately succeeded in proving that the normal scattering of a nearly vertical jet is due to the rebound of the drops when they come into collision with one another. Such collisions are inevitable in consequence of the different velocities acquired by the drops under the action of the capillary force, as they break away irregularly from the continuous portion of the jet. Even when the resolution is regularised by the action of external vibrations of suitable frequency, as in the beautiful experiments of Savart and Plateau, the drops must still come into contact before they reach the summit of their parabolic path. In the case of a continuous jet the "equation of continuity" shows that as the jet loses velocity in ascending, it must increase in section. When the stream consists of drops following the same path in single file, no such increase of section is possible, and then the constancy of the total stream requires a gradual approximation of the drops, which in the case of a nearly vertical direction of motion cannot stop short of actual contact. Regular vibration has, however, the effect of postponing the collisions and consequent scattering of the drops, and in the case of a direction of motion less nearly vertical may prevent them altogether.

Under moderate electrical influence there is no material change in the resolution into drops, nor in the subsequent motion of the drops up to the moment of collision. The difference begins here. Instead of rebounding after collision, as the unelectrified drops of clean water generally or always do, the electrified drops coalesce, and thus the jet is no longer scattered about. When the electrical influence is more powerful, the repulsion between the drops is sufficient to prevent actual contact, and then of course there is no opportunity for amalgamation.

\footnotetext{
1 "'The Influence of Electricity cn Colliding Water Drops." Paper read
} at the Royal Society by Lord Rayleigh, F.R.S. 
These experiments may be repeated with extreme ease and with hardly any apparatus. The diameter of the jet may be about $\frac{x}{20}$ inch, and may be obtained either from a hole in a thin plate or from a drawn-out glass tube. I have generally employed a piece of glass tube fitted at the end with a perforated tin plate, and connected with a tap by india-rubber tubing. The pressure may be such as to cause the jet to rise eighteen or twenty-four inches, or even more. A single passage of a rod of gutta-percha, or of sealing-wax, along the sleeve of the coat is sufficient to produce the effect. The seat of sensitiveness may be investigated by exciting the extreme tip only of a glass rod, which is then held in succession to the root of the jet and to the place of resolution into drop;. An effect is observed in the latter but not in the former position. Care must be taken to use an electrification so feeble as to require close proximity for its operation, otherwise the discrimination of the positions will not be distinct.

The behaviour of the colliding drops becomes apparent under instantaneous illumination. I have employed sparks from an inductorium, whose secondary terminals were connected with the coatings of a Leyden jar. The jet should be situated between the sparks and the eye, and the observation is facilitated by a piece of ground glass held a little beyond the jet, so as to diffuse the light; or the shadow of the jet may be received on the ground glass, which is then held as close as possible on the side towards the observer.

If the jet be supplied from an insulated vessel, the coalescence of colliding drops continues for a time after the removal of the influencing body. This is a consequence of the electrification of the vessel. If the electrified body be held for a time pretty close to the jet, and be then gradually withdrawn, a point may be found where the rebound of colliding drops is re-established. A small motion to or from the jet, or a discharge of the vessel by contact of the finger, again induces coalescence.

Although in these experiments the charges on the colliding drops are undoubtedly of the same name, it appeared to me very improbable that the result of contact of $t$ wo equal drops, situated in the open, could be affected by any strictly equal electrifications. At the same time an opposite opinion makes the phenomena turn tipon the very small differences of electrification due either to irregularities in the drops or to differences of situation, and is at first difficult of acceptance in view of the efficiency of such very feeble electric forces. Fortunately I am able to bring forward additional evidence bearing upon this point.

When two horizontal jets issue from neighbouring holes in a thin plate, they come into collision, for a reason that I need not now stop to explain, and after contact they frequently rebound from one another without amalgamation. This observation, which I suppose must have been made before, allowed me to investigate the effect of a passage of electricity across two contiguous water surfaces. The jets that I employed were of about 1 inch in diameter, and issued under a moderate pressure ( 5 or 6 inches) from a large stoneware vessel. Below the place of rebound, but above that of resolution into drops, was placed a piece of insulated tin plate in connection with a length of gutta-percha-covered wire. The source of electricity was a very feebly excited electrophorus, whose cover was brought into contact with the free end of the insulated wire. When both jets played upon the tin plate the contact of the electrified cover had no effect in determining the union, but when only one jet washed the plate union instantly followed the communication of electricity, and this notwithstanding that the jets were already in communication through the vessel. The quantity of electricity required is so small that the cover would act three or even four times without being re-charged, although no precautions were taken to insulate the reservoir.

In subsequent experiments the colliding jets, about $\frac{8}{10} \delta$ inch in diameter, issued horizontally from similar glass nozzles, formed by drawing out a piece of glass tubing and dividing it with a file at the narrowest part. One jet was supplied from the tap, and the other from the stoneware bottle placed upon an insulating stool. The sensitiveness to electricity was extraordinary. A piece of rubbed gutta-percha brought near the insulated bottle at once determined the coalescence of the jets. The influencing body being held still, it was possible to cause the jets again to rebound from one another, and then a small motion of the influencing body to or from the bottle again induced coalescence, but a lateral motion without effect. If an insulated wire be in connection with the contents of the bottle, similar effects are produced when the electritied body is moved in the neighbourbood of the free end of the wire. With care it is possib!e to bring the electrified body into the neighbourhood of the free end of the wire so slowly that no effect is produced; a sudden movement of withdrawal will then usually determine the coalescence.

Hitherto statical electricity has been spoken of ; but the electromotive force of even a single Grove cell is sufficient to produce these phenomena, though not with the same certainty. For this purpose one pole is connected through a contact key with the interior of the stoneware bottle, the other pole being to earth. If the fingers be slightly moistened, the body may be thrown into the circuit, apparently without diminution of effect. This perhaps ought not to surprise us, as in any case the electricity has to traverse several inches of a fine column of water. On the other hand, it appeared that most of the electromotive force of the Grove cell was necessary.

Further experiment showed that even the discharge of a condenser charged by a single Grove cell was sufficient to determine coalescence. Two condensers were used. successively; one belonging to an inductorium by Ladd, the other made by Elliott Brothers, and marked "Capacity $\frac{1}{2}$ Farad." Sometimes even the "residual charge", sufficed.

It must be understood that coalescence of the jets would sometimes ozcur in a capricious manner, without the action of electricity or other apparent cause. I have reason to believe that some, at-any rate, of these irregularities depended upon a want of cleanness in the water. The addition to the water of a very small quantity of soap makes the rebound of the jets impossible.

The last observation led me to examine the behaviour of a fine vertical jet of slightly soapy water: and I found, as I had expected, that no scatterino took place. Under these circumstances the approach of a moderately electrified body is without effect, but a more powerful influence scatters the drop as usual. The apparent coherence of a jet of water when the orifice is oiled was observed by Fuchs, and appears to have been always attributed to a diminution of adhesion between the jet and the walls of the orifice.

Some further details on this subject, and other investigations respecting the phenomena of jets, are reserved for another communication, which I hope soon to be able to present to the Royal Society; but I cannot close without indicating the probable application to meteorology of the facts already mentioned. It is obvious that the formation of rain must depend very materially upon the consequences of encounters between cloud particles. If encounters do not lead to contacts, or if contacts result in rebounds, the particles remain of the same size as before ; but, if the issue be collescence, the bigger drops must rapidly increase in size and be precipitated as rain. Now, from what has appeared above, we have every reason to suppose that the results of an encounter will be different according to the electrical condition of the particles, and we may thus anticipate an explanation of the remarkable but hitherto mysterious connection between rain and electrical manifestations. 